\title{
Optical waveform monitoring based on a free-running mode-locked femtosecond fibre laser and four-wave mixing in a highly nonlinear fibre
}

\author{
Y. LIU1 ${ }^{1}$, J.-G. ZHANG ${ }^{* 2}$, and D. TANG ${ }^{3}$ \\ ${ }^{1}$ State Key Laboratory of Transient Optics and Photonics, Xi' an Institute of Optics and Precision Mechanics, \\ Chinese Academy of Sciences, Xi'an, Shaanxi 710119, China \\ ${ }^{2}$ Biomedical Engineering and Communications Research Group, School of Engineering, London South Bank \\ University, 103 Borough Road, London SE1 0AA, UK \\ ${ }^{3}$ Southwest China Research Institute of Electronic Equipment, Chengdu 610036, China
}

Optical sampling based on ultrafast optical nonlinearities is a useful technique to monitor the waveforms of ultrashort optical pulses. In this paper, we present a new implementation of optical waveform sampling systems by employing our newly constructed free-running mode-locked fibre laser with a tunable repetition rate and a low timing jitter, an all-optical waveform sampler with a highly nonlinear fibre (HNLF), and our developed computer algorithm for optical waveform display and measurement, respectively. Using a femtosecond fibre laser to generate the highly stable optical sampling pulses and exploiting the four-wave mixing effect in a $100 \mathrm{~m}$-long $\mathrm{HNLF}$, we successfully demonstrate the all-optical waveform sampling of a 10GHz optical clock pulse sequence with a pulse width of $1.8 \mathrm{ps}$ and a 80Gbit/s optical data signal, respectively. The experimental results show that waveforms of the tested optical pulse signals are accurately reproduced with a pulse width of 2.0 ps. This corresponds to a temporal resolution of $0.87 \mathrm{ps}$ for optical waveform measurement. Moreover, the optical eye diagram of a 10Gbit/s optical data signal with a $1.8 \mathrm{ps}$ pulse width is also accurately measured by employing our developed optical sampling system.

Keywords: optical sampling, mode-locked fibre laser, four-wave mixing, highly nonlinear fibre, optical waveform monitoring, eye diagram measurement.

\section{Introduction}

Fast and accurate characterization of ultrashort optical pulses is often required by, for example, the study of picosecond/ subpicosecond pulsed lasers, ultrahigh-speed optical transmitters, light pulse propagation in various optical media (including optical fibres), and ultrafast all-optical signal processing, respectively. A simple application of pulse evaluation is to monitor the waveforms of ultrashort optical pulses and to measure the full-width at half-maximum, rise and fall times of such a pulse. To achieve this goal, we can use three conventional measurement approaches. One is called the autocorrelation measurement which has a symmetric response function, thus preventing it from the evaluation of rise and fall times for optical pulses and the analyses of asymmetric optical waveforms and/or optical data signals modulated with pseudorandom binary sequence (PRBS) [1,2], respectively. The second scheme is referred to as the "opto-electronic oscilloscope" equipment that consists of a conven-

*e-mail: zhangja@1sbu.ac.uk, jian-guo-zhang@126.com tional wideband electronic oscilloscope and an ultrafast photodetector connected in series. This approach, however, suffers from the bandwidth limitation imposed by optical-to-electrical conversion and the associated electronic circuits. At present, the bandwidth of state-of-the-art electronic oscilloscopes is restricted to $100 \mathrm{GHz}$ and the commercially available photodetectors can have a bandwidth beyond 100 $\mathrm{GHz}$, thus limiting the measurement bandwidth of the resulting "opto-electronic oscilloscopes" to be about $100 \mathrm{GHz}$. Moreover, the optical front-end of such an oscilloscope is based on an ultrafast photodetector which, in turn, leads to a much poorer optical receiver sensitivity than that based on a low-speed photodetector (e.g., $\leq 100 \mathrm{MHz}$ ). The last approach is to use a streak camera which, however, has a low sensitivity at the wavelength around $1550 \mathrm{~nm}$ [1-3], and, therefore, precludes the monitoring of optical eye diagrams. Consequently, a new type of optical waveform measurement systems needs to be developed, which should use the all-optical waveform sampling based on ultrafast optical nonlinearities to ensure a very wide measurement bandwidth up to several hundreds of gigahertz or a few terahertz $[1,2]$. This, 
in turn, corresponds to a subpicosecond temporal resolution for the developed waveform measurement system.

All-optical waveform sampling $[1,2]$ is a very useful technique which can be employed to directly measure the waveforms of ultrashort optical pulses generated by ultrafast lasers [1,4,5] or propagating in optical fibres [6] (or waveguides) and the eye diagrams of ultrahigh-speed optical data signals in optical fibre communications [5-7], respectively. This is achieved by using optical signal processing to remove the bandwidth limitation of electronic circuits as encountered in traditional electronic oscilloscopes. According to hardware configurations, optical waveform sampling systems can be generally divided into two major categories [8,9], i.e., synchronous sampling and asynchronous sampling, respectively. The synchronous optical sampling scheme is capable of accurately rebuilding the waveforms or eye diagrams of optical data signals by employing photonic clock-recovery circuits $[6,9]$. In doing so, this results in the increase of both system complexity and cost. In contrast, asynchronous optical sampling systems do not need the expensive clock-recovery circuits, thus having the advantages of both simple hardware structure and low system cost. However, the latter suffers from the inaccurate measurement of a Q-factor [10,11]. To solve these problems, a software-synchronized optical sampling technique was proposed by Westlund and Andrekson et al. $[1,10,11]$, which uses the software algorithm based on the Fourier transform of the sampled data and the eye-diagram timing drift to provide the synchronization information required for accurate Q-factor measurement and waveform/ eye-diagram monitoring of ultrahigh-speed optical data signals.

At present, all-optical waveform sampling is normally implemented by exploiting the nonlinear optical effects in optical media such as optical fibres $[1,2,7]$, waveguides $[3,12,13]$ and crystals $[4,5]$. These include the second-order susceptibility $\chi^{2}$ in nonlinear crystals (e.g., $\mathrm{KTiOPO}_{4}(\mathrm{KTP})[14,15]$ and periodically poled $\mathrm{LiNbO}_{3}$ (PPLN) crystals $[4,5]$ ) and the third-order susceptibility $\chi^{3}$ (e.g., cross-phase modulation and four-wave mixing) in nonlinear optical fibres $[1,2,16]$, semiconductor optical amplifiers (SOAs) [3,17], and silicon nanowire [12] or chalcogenide planar waveguide [13], respectively. Nevertheless, those schemes can have their individual advantages and respective drawbacks in terms of operational speed, wavelength range, manufacturing cost, complexity and physical size of the resulting optical waveform samplers (also called sampling gates). For example, the use of sun-frequency generation (SFG) in nonlinear crystals allows to implement ultrafast optical samplers which are capable of measuring the optical data signals up to $640 \mathrm{Gbit} / \mathrm{s}$ [5], while the resulting samplers are normally bulky and their operational wavelength range is also restricted by the phase-matching condition of nonlinear crystals [7,9]. Although a PPLN crystal can be employed to sample optical signal waveforms with higher temporal resolution and larger SFG conversion efficiency than the KTP crystal, a drawback of the former is the requirement for an accurate control of operation temperature $[4,13]$ at the expense of increasing the system complexity and cost. On the other hand, if the SOA-based waveform samplers are used, their operational speed can be ultimately limited by the relatively long gain recovery time in SOAs, and the amplified spontaneous emission noise of a SOA can also cause a degradation of signal-to-noise ratio for the optically sampled signal. Moreover, optical samplers based on advanced silicon nanowire and chalcogenide planar waveguide are promising for optical waveform monitoring, as they are capable of ultrahigh-speed operations and integrated implementation on a single photonic chip $[12,13]$. However, these chip-scale optical samplers require a sophisticated fabrication process which can prevent them from practical applications nowadays. Moreover, the silicon and chalcogenide waveguides also possess a large coupling loss when they are connected with fibre-based devices in photonic communication systems and networks. For all-optical waveform sampling applications, it is clear that there is a trade-off between optical sampling performance and system cost. Among those reported implementation schemes, fibre-based optical waveform sampling systems are very useful for engineering design and practical applications nowadays. This is because silica optical fibres enable massive production with mature technology and low cost, besides their high operation reliability. Moreover, optical waveform sampling systems based on silica fibres have a low complexity for implementation and also have a low coupling loss with optical/optoelectronic devices having pigtail fibres which are widely used in optical fibre communication systems/networks. Moreover, silica fibres possess the femtosecond response time of Kerr nonlinearity which, in turn, allows to realize ultrafast optical waveform samplers for monitoring optical data signals at $640 \mathrm{Gbit} / \mathrm{s}$ and beyond [1]. Nevertheless, conventional single-mode fibres are inadequate for optical sampling applications, because the small nonlinear refractive index of such fibres makes a long interactive length or high input power necessary for obtaining sufficient nonlinear effects. To overcome this difficulty, a highly nonlinear fibre (HNLF) can be utilized to effectively shorten the fibre length or lower down input power required by optical waveform samplers [1]. In doing so, this makes fibre-based waveform samplers more attractive for practical applications in terms of physical size, operational stability (affected by environmental disturbances), implementation complexity, optical sampling performance, and manufacture cost of the resulting waveform samplers. Furthermore, the use of HNLF-based ultrafast sampler and free-running femtosecond fibre laser allows the implementation of a cost-effective optical waveform sampling system, as we will report in Sect. 3 of this paper.

Four-wave mixing (FWM) $[1,2,16]$ and cross-phase modulation (XPM) [7] in HNLFs have been studied for all-optical waveform sampling applications, due to their low cost and 
easy implementation. Compared to XPM, FWM has the characteristics of phase information preservation and transparency to modulation formats of optical data signals. This, in turn, makes FWM-based optical sampling systems very suitable for practical applications. However, the reported work on fibre-based optical waveform sampling has mainly focused on HNLF-based optical sampling gates [1,2,7], software-synchronization technique for all-optical sampling of ultrashort optical pulse waveforms $[10,11,16]$, and the associated system performance $[1,2,18]$. From the viewpoint of engineering applications, there is a need of systematic design for constructing the whole optical waveform sampling system. In particular, its feasible implementation based on commercially available photonic devices is highly desired by optics/optical telecom researchers who plan to self-build up cost-effective optical waveform sampling systems at their respective laboratories rather than purchasing such sampling systems which are commercially available only from a few companies worldwide. Moreover, the practical selection of a repetition rate for optical sampling sources also needs to be considered. In this paper, we investigate into the new implementation of a cost-effective optical sampling system with subpicosecond temporal resolution for optical waveform monitoring. This is achieved by using our constructed low-timing-jitter, passively mode-locked femtosecond fibre laser with a tunable repetition rate, an all-optical waveform sampler using FWM in a 100m-long HNLF, and our developed computer algorithm for optical waveform display and measurement based on the software-synchronized optical sampling technique [10,11], respectively. The procedure for implementing such a waveform sampling system is also discussed in detail. In the experiments, we successfully demonstrate the all-optical waveform sampling of a $10 \mathrm{GHz}$ optical clock pulse sequence with a pulse width of $1.8 \mathrm{ps}$ and a $80 \mathrm{Gbit} / \mathrm{s}$ optical data signal, respectively. The optical pulse waveforms are then reproduced by using digital signal processing and a software-synchronized optical sampling method, with which the associated pulse parameters can be obtained from a computer. We also use this optical sam- pling system to monitor the eye diagram of a $10 \mathrm{Gbit} / \mathrm{s}$ optical data signal modulated with a PRBS in the experiment.

\section{Optical waveform monitoring by flexible optical sampling}

A typical optical waveform sampling system consists of an optical sampling pulse source, an all-optical sampling gate (i.e., sampler), an "optical-to-electrical (O/E) and analogue-to-digital (A/D) conversion" unit, and an "electronic data processing and waveform display" unit, respectively, as illustrated in Fig. 1. Its basic operation principle can be explained with the aid of Fig. 2. To properly sample an optical pulse sequence or data signal, the width of optical sampling pulses should be narrower than that of the tested optical pulse signal. Moreover, the jitter of an optical sampling pulse source should be kept as low as possible in order to accurately sample the ultrashort optical pulses under test. This in turn requires the use of a low-timing-jitter, passively mode-locked femtosecond fibre laser to generate a highly stable train of optical sampling pulses. To correctly acquire the waveform information of the optical signal under test, the repetition frequency $f_{s}$ of a periodic optical sampling pulse train should be determined according to the following relationship $[2,10,11]$

$$
B=M f_{s}+\Delta f,
$$

where $B$ denotes the repetition rate of the tested periodic optical clock-pulse train or the bit rate of the tested optical data signal, the integer $M$ corresponds to the bit rate reduction factor, and $\Delta f$ is the offset frequency between $B$ and $M f_{s}$. The parameter $\Delta f$ determines the bit-slot scanning rate for optical waveform sampling $[10,11]$, which can be properly adjusted to meet the requirement of bit-rate flexible operations. In fact, this requires to appropriately set the repetition frequency of an optical sampling pulse source. Consequently, it is crucial to use a passively mode-locked femtosecond fibre laser with tunable repetition frequency and low timing jitter in the optical waveform sampling system.

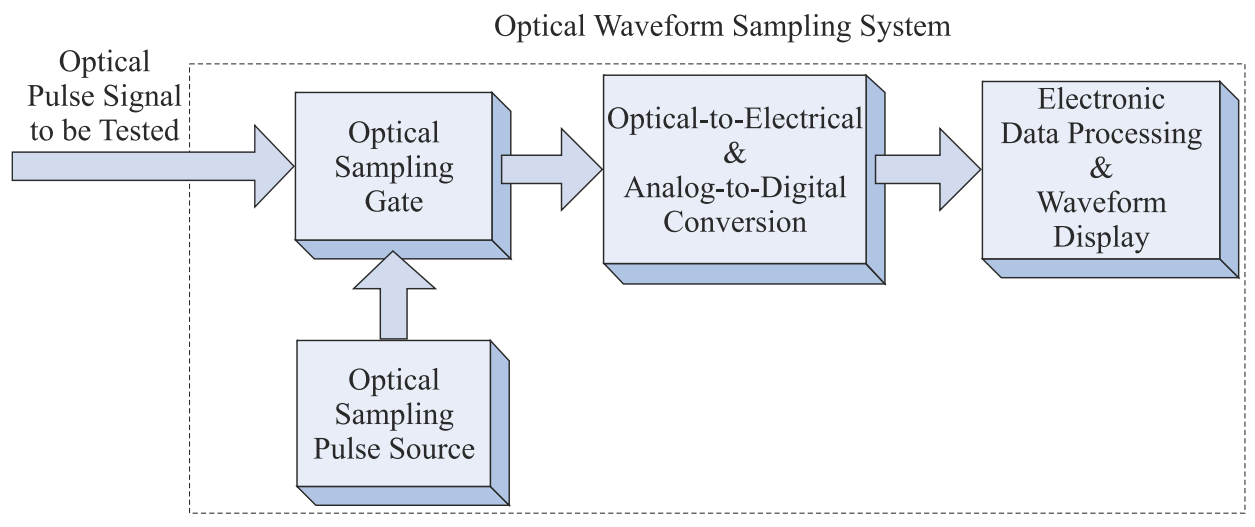

Fig. 1. Functional block diagram of a typical optical waveform sampling system. 
Original Optical Data Pulse Signal

(1)

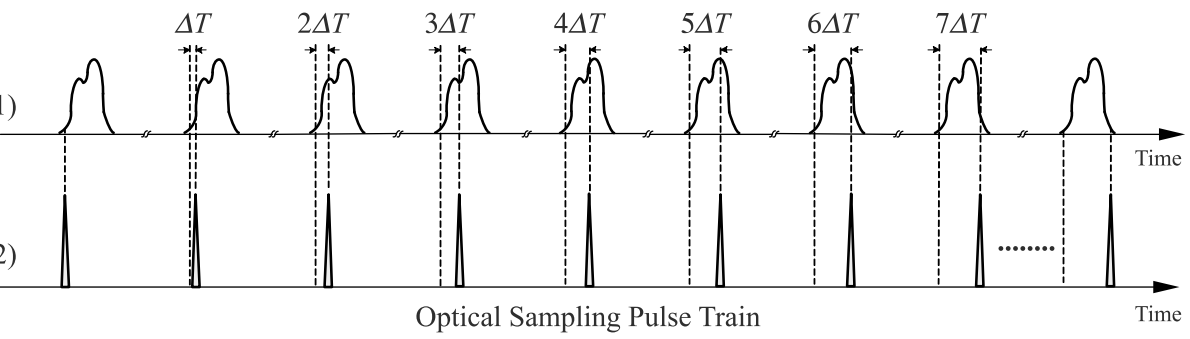

(3)

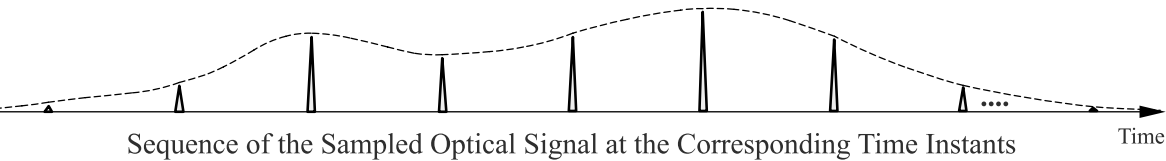

(4)

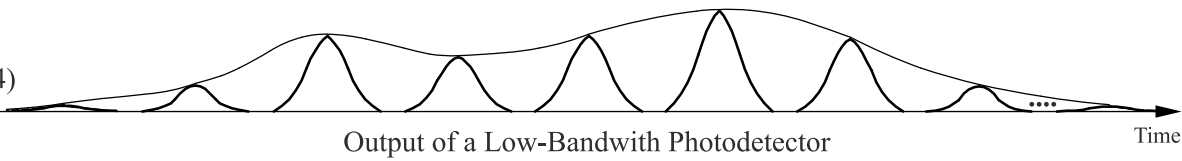

(5)

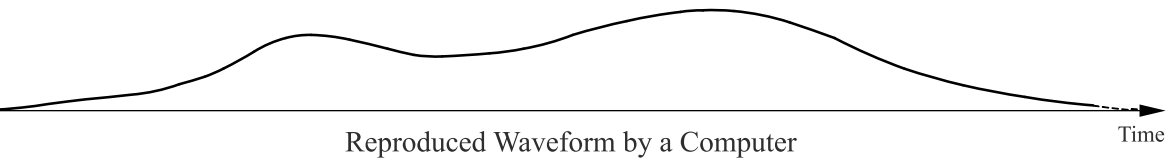

(a)
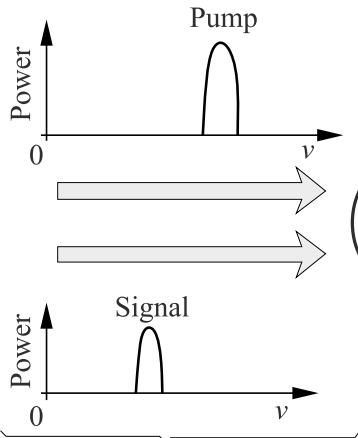

Spectra of Input Lights to a HNLF

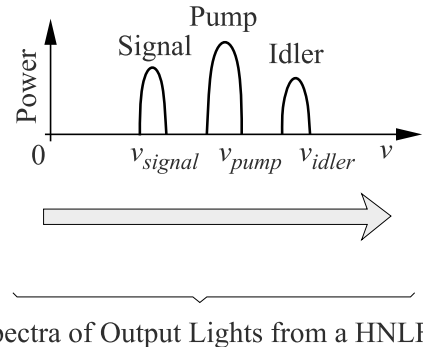

(b)

Fig. 2. Illustrations of (a) optical waveform sampling in the time domain and (b) the associated FWM process in the frequency domain, respectively.

FWM in a HNLF can be practically employed to realize the process of ultrafast optical waveform sampling, in which a periodic optical sampling pulse train is used as the intense pump wave to cause the power-dependent refractive index of the optical fibre. When both pump and signal lights of distinct optical frequencies are launched into an optical sampling gate based on the HNLF, under the phase-matching condition, the FWM interaction of pump and signal waves results in the generation of a sampled signal wave, called the idler, at a new optical frequency $v_{\text {idler }}$ [see Fig. 2(b)] $[1,18]$, i.e.

$$
v_{\text {idler }}=2 v_{\text {pump }}-v_{\text {signal }},
$$

where $v_{\text {pump }}$ and $v_{\text {signal }}$ are optical frequencies of pump and signal lights, respectively. In this way, the idler can be extracted by optical band-pass filtering at the HNLF output of an optical sampling gate, followed by the optical-to-electrical conversion and further data processing, as will be described subsequently. If the parameter $\Delta f$ in Eq. (1) is nonzero, the sampling time instants would appear in the different time locations with respect to a normalized signal bit period [see Fig. 2(a)]. For a repetitive optical waveform, this is equivalent to the creation of sampling time instants having a step $\Delta T$ within each signal bit period $[1,10,19]$

$$
\Delta T=\frac{1}{f_{s}}-\frac{M}{B} .
$$

At an optical sampling gate, the optical signal waveform is acquired by means of those discrete samples which are carried 
on top of the uniformly-spaced optical pulses at an interval of $1 / f_{s}$, as shown in Fig. 2(a) (3). After photodetection, the envelope of the detected pulses, which is indicated by the dashed line in Fig. 2(a) (4), is analogous to the waveform of the original optical signal under test except for the time scale. Such electronic pulses at a low pulse rate of $f_{s}$ must be fed into an A/D converter, so that a computer can be used to efficiently process the electronic data in order to obtain the synchronization information of the acquired samples, time step $\Delta T$ of optical waveform sampling, pulse width, rise or fall time, and bit period of an optical signal, as well as other time-related signal parameters. In doing so, the computer can reconstruct the complete optical signal waveform for periodic optical pulse trains (or repetitive optical bit patterns) or the eye diagram for the tested optical data signal that is modulated with a pseudorandom bit sequence. In effect, this is achieved by using the software-based synchronization technique (proposed by Westlund et al. $[1,10$, 11]) with no need of a clock recovery circuit.

For equivalent-time optical sampling with $f_{s}<<B$, the precision in the reconstruction of an optical waveform is mainly dependent on a time step $\Delta T$ given by Eq. (3). A higher-precision waveform is reproduced when the value of $\Delta T$ is chosen to be smaller at the expense of a longer time for waveform reconstruction or vice versa, as illustrated in Fig. 2. This, in turn, requires the adjustment of $f_{s}$ based on Eqs. (1) and (3) for a given $B$. In practice, the optical data signals from a variety of optical fibre communication systems can have the different bit rates. Moreover, for the purpose of laboratory research, various pulsed lasers (e.g., gain-switched or mode-locked lasers) under the test can also generate the periodic optical clock-pulse trains with distinct repetition rates (or even a tunable repetition rate in the gain-switching operation [20]). The measurement of such signal or clock-pulse waveforms normally requires to adjust $f_{s}$ in order to keep a constant $\Delta T$ or to achieve a desirable precision which can be needed by users to meet their specific test requirements. To achieve this aim, we should use an optical sampling pulse source with a changeable repetition rate to flexibly generate a train of stable optical sampling pulses in order to obtain the desired $\Delta T$ based on Eq. (3). Such a requirement can be satisfied by using our constructed low-timing-jitter, free-running passively mode-locked femtosecond fibre laser with tunable repetition rate and high operation stability [21]. This is regarded as an advantage of our designed optical waveform sampling system.

\section{Experimental setup and measurements}

Based on the above discussions, we can see that the implementation of a high-performance optical waveform sampling system requires the use of (1) highly reliable optical sampling source, (2) low-complexity optical waveform sampling gate, (3) cost-effective photodetection and A/D conversion modules, and (4) efficient computer algorithms for software-based synchronization, optical waveform/eye-diagram display and parameter measurements, respectively. The experimental setup is shown in Fig. 3. In the following, we describe the

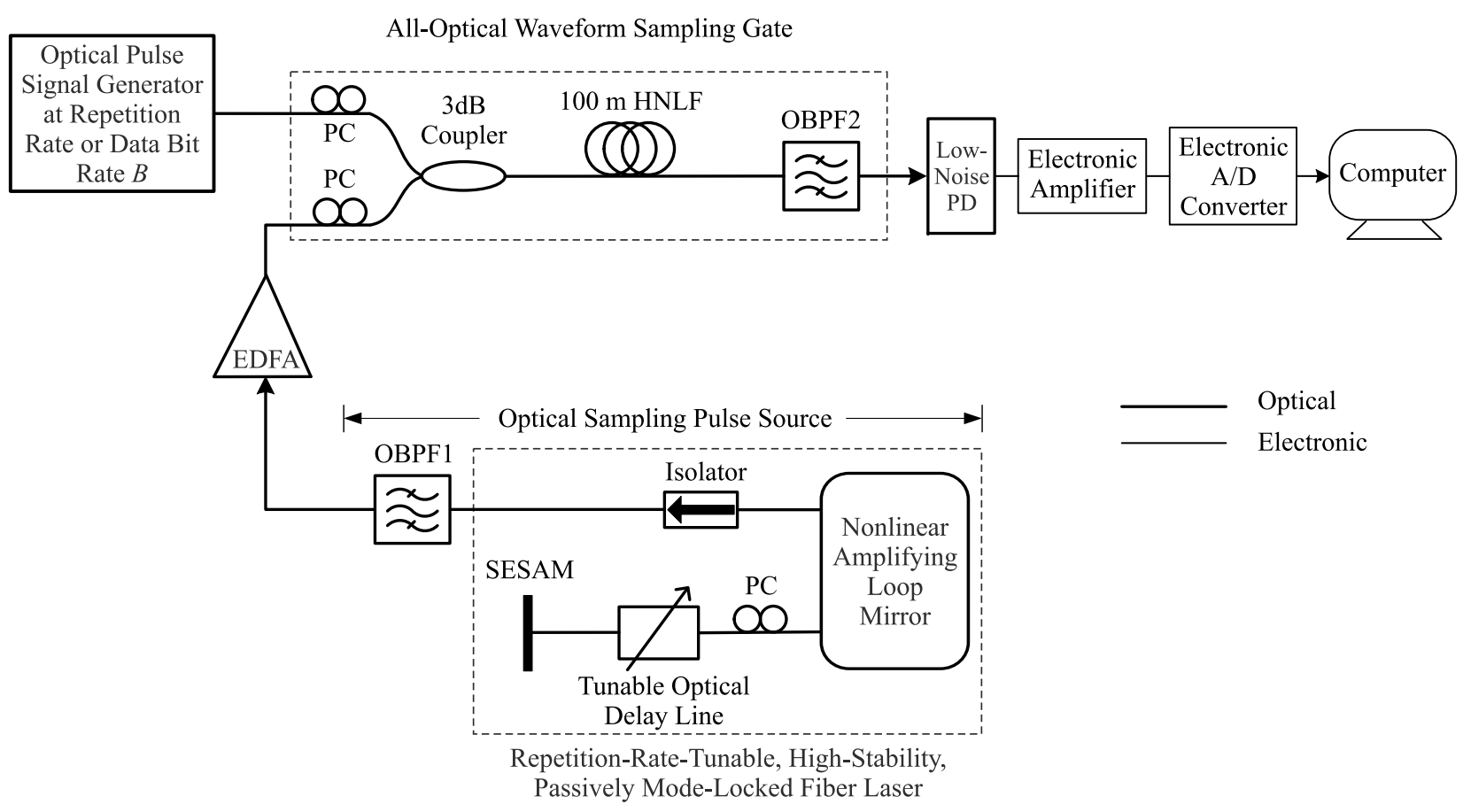

Fig. 3. Experimental setup of an optical waveform sampling system with a passively mode-locked femtosecond fibre laser and a FWM-based optical waveform sampling gate. 
design procedure for a fibre-based waveform sampling system and the experiment results associated with optical waveform/ eye-diagram monitoring.

For an optical hardware implementation in a cost-effective manner, the constructed optical sampling system uses a free-running passively mode-locked fibre laser (with tunable repetition rate and low timing jitter) as the optical sampling pulse source and a FWM-based optical gate with a $100 \mathrm{~m}$-long HNLF as the ultrafast waveform sampler, respectively. In our experiment, the optical signal under test is produced by an optical pulse signal generator which substantially uses an actively mode-locked semiconductor laser ( $\mathrm{u} 2 \mathrm{t}$ model number TMLL 1550) to provide a stream of original optical clock pulses at a repetition frequency of $10 \mathrm{GHz}$ and a central wavelength of $1554 \mathrm{~nm}$ for data modulation and optical time-division multiplexing (OTDM), as shown in Figs. 4(a) and 4(b), respectively. The width of the obtained optical clock pulses is 1.8 ps measured by an autocorrelator with resolution $<5 \mathrm{fs}$ (see Fig. 5). For the purpose of eye diagram monitoring at 10 Gbit/s, a $\mathrm{LiNbO}_{3}$ intensity modulator is employed to on-off modulate the output clock pulse stream of this actively mode-locked semiconductor laser by using the $10 \mathrm{Gbit} / \mathrm{s}$ electronic PRBS data signal that is obtained from a bit-error-rate (BER) tester. Both actively mode-locked semiconductor laser and BER tester are synchronized with a common RF clock signal source, as shown in Fig. 4(a). Consequently, a 10 Gbit/s optical PRBS data signal is generated with a pulse width of $1.8 \mathrm{ps}$. In the experiment, we also need to measure the waveform of a $80 \mathrm{Gbit} / \mathrm{s}$ optical pulse signal which can be formed by opti- cally time multiplexing either a $10 \mathrm{GHz}$ optical clock pulse sequence or a $10 \mathrm{Gbit} / \mathrm{s}$ optical data signal. For the sake of simplicity, an OTDM multiplexer is inserted at the output of an actively mode-locked semiconductor laser without employing a $\mathrm{LiNbO}_{3}$ intensity modulator in between, as shown in Fig. 4(b). To ensure the sufficient power for the generated OTDM signal or optical PRBS data signal, an erbium-doped fibre amplifier (EDFA) is employed at the output of an actively mode-locked semiconductor laser or an optical intensity modulator (see Fig. 4). The OTDM multiplexer simply consists of $1 \times 4$ and $4 \times 1$ optical couplers with single-mode fibre (SMF) pigtails, 3 tunable optical delay lines (T-ODL's) with SMF pigtails, and an optical reference path containing a variable optical attenuator (VOA) with two SMF pigtails of appropriate lengths, respectively. Its operation is based on three steps: (1) split an input 1.8 ps optical pulse into four parts, (2) interleave four split pulses through proper optical delays in order to fit into four consecutive time slots of which each has a width of $12.5 \mathrm{ps}$, and (3) combine those four pulses to form an optical signal pattern containing four successive pulses at $80 \mathrm{Gbit} / \mathrm{s}$. Therefore, a $80 \mathrm{Gbit} / \mathrm{s}$ optical data signal with the fixed optical pulse pattern of " 11110000 " and return-to-zero (RZ) format is obtained from this OTDM multiplexer when its input signal is a $10 \mathrm{GHz}$ optical clock pulse stream. The waveform of the resulting $80 \mathrm{Gbit} / \mathrm{s}$ OTDM signal is also monitored by employing a $70 \mathrm{GHz}$ electronic sampling oscilloscope (Tektronix TDS8200) connected with a $70 \mathrm{GHz}$ photodiode (PD, u2t XPDV3120R) at the output of the OTDM multiplexer, as shown in Fig. 6. Note that the pulse waveform

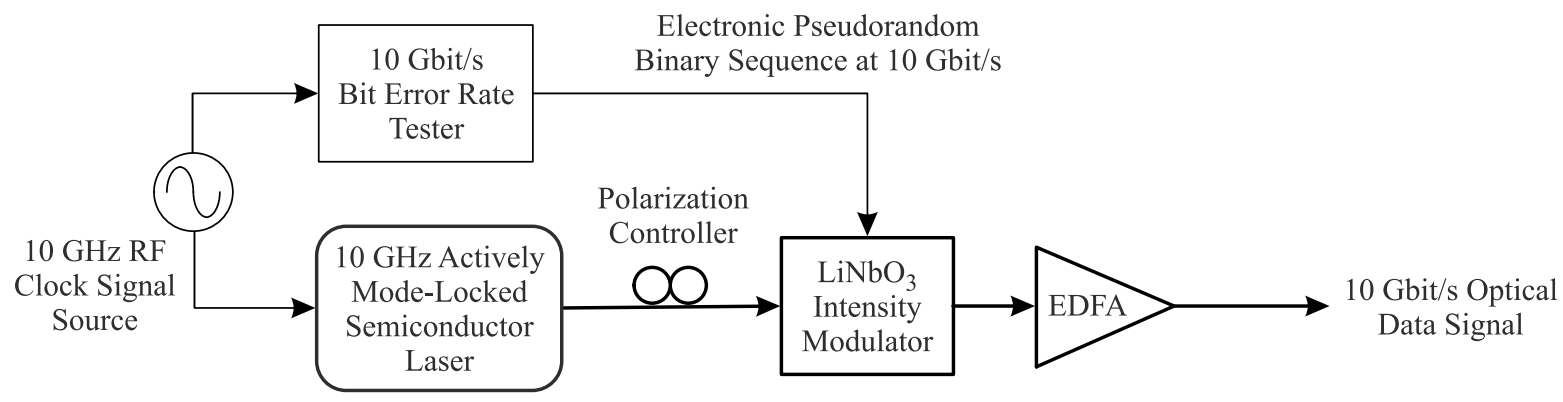

(a)

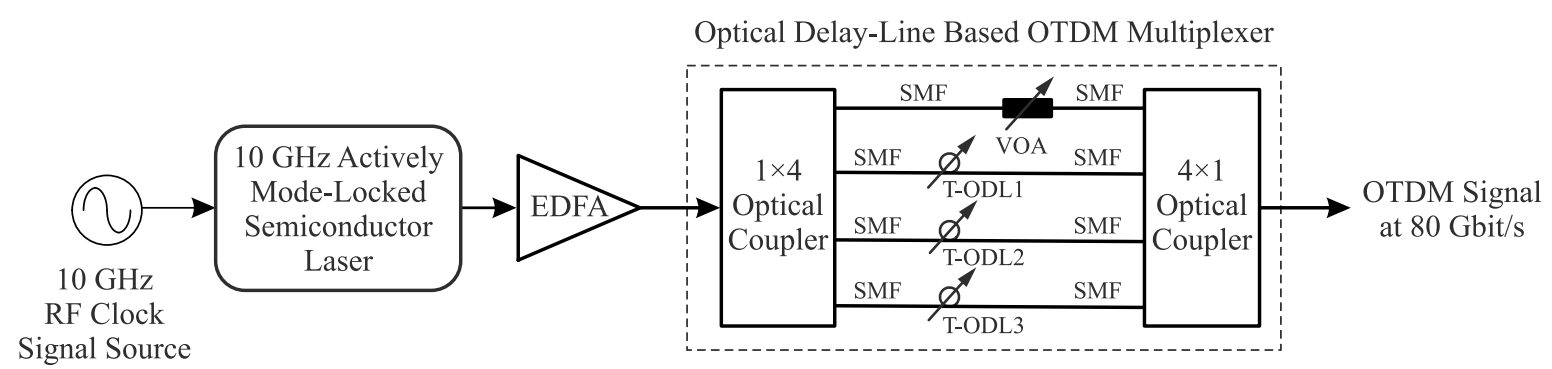

(b)

Fig. 4. Experimental setup of (a) $10 \mathrm{Gbit} / \mathrm{s}$ optical PRBS pulse signal generator and (b) $80 \mathrm{Gbit} / \mathrm{s}$ OTDM pulse signal generator. 
Optical waveform monitoring based on a free-running mode-locked femtosecond fibre laser and four-wave mixing...

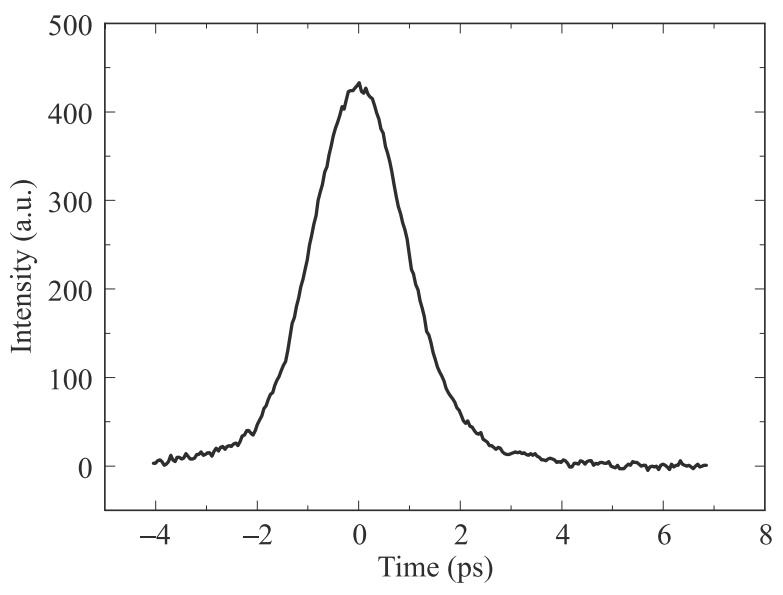

Fig. 5. Autocorrelation trace of an optical clock pulse generated by a $10 \mathrm{GHz}$ actively mode-locked semiconductor laser.

of the $80 \mathrm{Gbit} / \mathrm{s}$ signal with pulse width of about $1.8 \mathrm{ps}$ is not resolved due to the limited bandwidth of $\sim 70 \mathrm{GHz}$ of a conventional optoelectronic measurement system. This problem would be effectively solved if we use an optical waveform sampling system, as will be discussed subsequently.

To accurately sample the waveforms of optical signals under test, we should use an optical sampling pulse source that is capable of producing a stream of ultrashort optical pulses with low timing jitter and high stability. We have recently developed a low-timing-jitter, stretched-pulse passively mode-locked femtosecond fibre laser which has a tunable repetition rate over a range from $49.65 \mathrm{MHz}$ to $50.47 \mathrm{MHz}$ and a timing jitter less than 75 fs over a frequency range of $100 \mathrm{~Hz}$ to $100 \mathrm{kHz}$ [21], respectively. The generated stream of femtosecond optical pulses has a central wavelength of $1544 \mathrm{~nm}$. Moreover, this mode-locked fibre laser can keep the mode-locking operation continuously over 10 hours without the adjustment of any optical device in the laser cavity. In our previous experiments, such a fibre laser was able to achieve the self-starting, passive mode locking at a low pump power of $29 \mathrm{~mW}$ for stable generation of femtosecond optical pulses [22], when a tunable optical delay line was not used in the laser cavity. A distinguishing feature of tunable repetition rate and low timing jitter for our developed passively mode-locked fibre laser allows the bit-rate flexible operation in a cost-effective manner to meet the requirements of various users for optical waveform monitoring applications. Thus, it is expected that our designed optical waveform sampling system can have a high operational flexibility. In practice, the optical sampling pulse train with a repetition rate of about $50 \mathrm{MHz}$ is obtained by using a tunable optical band-pass filter (OBPF) with a $3 \mathrm{~dB}$

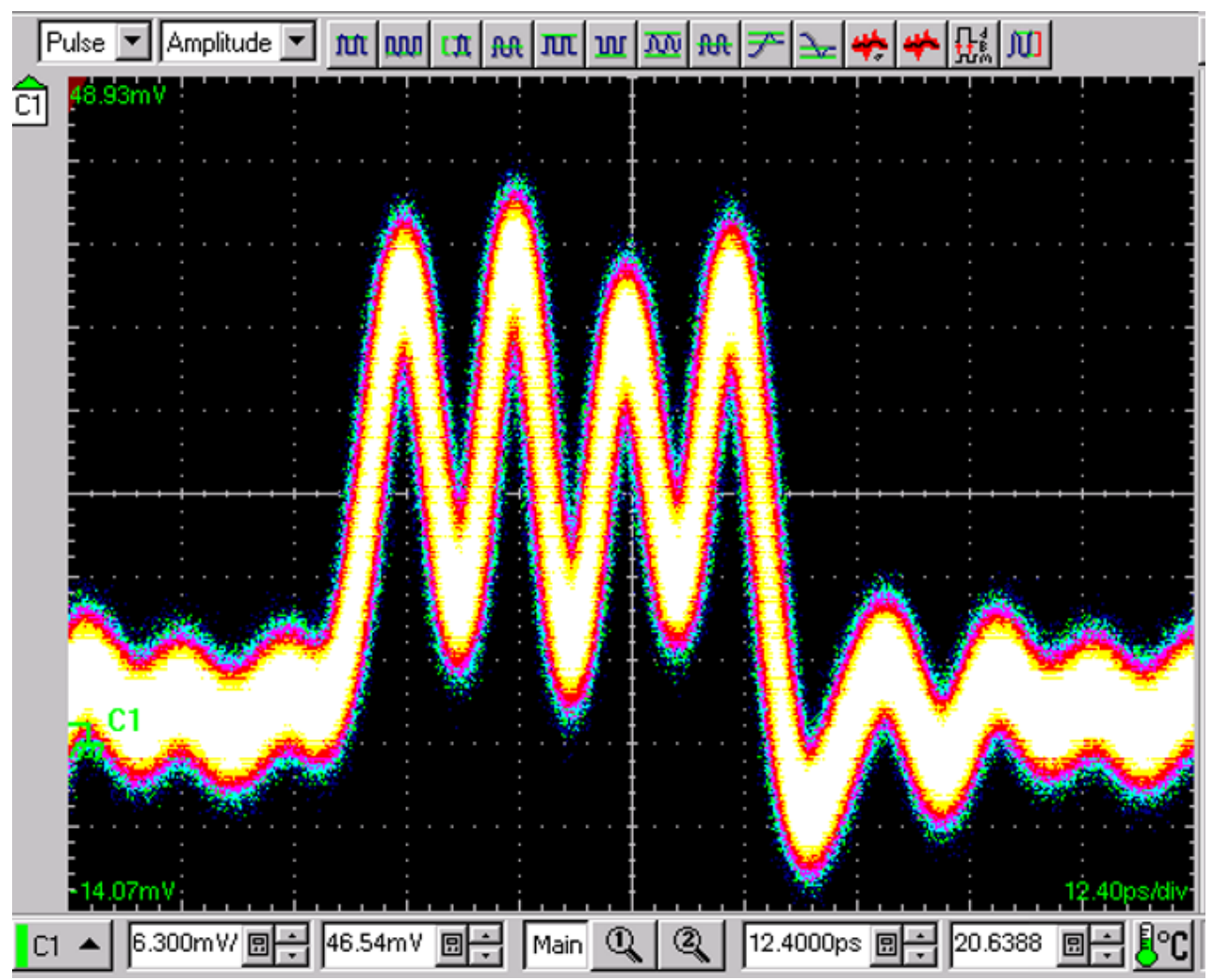

Fig. 6. $80 \mathrm{Gbit} / \mathrm{s}$ signal waveform with 4 adjacent optical pulses measured by a $70 \mathrm{GHz}$ electronic oscilloscope cascaded with a $70 \mathrm{GHz}$ photodiode at the output of an OTDM multiplexer. 
bandwidth of $2.5 \mathrm{~nm}$ to reduce the spectral width of an original femtosecond optical pulse train at the output of this mode-locked fibre laser, so that the width of the used optical sampling pulses is increased to about $1 \mathrm{ps}$ and is still adequate for measuring the optical signal waveform having a pulse width of $1.8 \mathrm{ps}$ as will be reported subsequently. In doing so, this can substantially alleviate the effect of group-velocity dispersion (GVD) on optical sampling pulses while propagating in the $100 \mathrm{~m}$-long HNLF of an optical waveform sampling gate. With a narrower spectrum for the sampling-pulse light, the walk-off (due to the GVD) between a sampling pulse and a signal pulse can be also reduced in a 100 m-long HNLF, because a smaller separation between the optical spectra of sampling and signal lights is allowed to use then the case with original optical sampling pulses possessing the pulse width of a few hundred femtoseconds. As a result, the temporal resolution of the designed waveform sampling gate can be improved. Moreover, the use of a wider optical sampling pulse can result in the less effect of self-phase modulation on the optical-nonlinearity-based sampling of optical data signal waveforms in a HNLF [1]. This can also lead to enhancing the performance of an all-optical waveform sampling gate.

At present, the use of highly nonlinear fibres can be treated as a feasible solution to the implementation of ultrafast waveform sampling gates in terms of cost and commercial availability. The $100 \mathrm{~m}$-long HNLF used in our experiment has a nonlinear coefficient of $10.5 \mathrm{~W}^{-1} \mathrm{~km}^{-1}$, a dispersion of $0.04 \mathrm{ps} /(\mathrm{nm} \mathrm{km})$ at $1550 \mathrm{~nm}$, a zero dispersion wavelength of $1546 \mathrm{~nm}$, and a total loss of $0.43 \mathrm{~dB}$. A reel of $100 \mathrm{~m} \mathrm{HNLF}$ is relatively compact, as shown in Fig. 7 where a normal-size pen is also illustrated in the lower right corner to compare with the outside reel diameter. At the input of a HNLF-based waveform sampling gate, two polarization controllers (PCs) are employed to adjust the polarization states of sampling and data signal lights so as to optimize the FWM conversion efficiency in a HNLF, respectively. Here the sampling pulse light serves as a pump in the FWM process. Then optical sampling pulse train and optical data signal are combined by a $3 \mathrm{~dB}$ optical coupler before they are fed into a $100 \mathrm{~m}$-long HNLF, as illustrated in Fig. 3. Clearly, the wavelengths of sampling light at $1544 \mathrm{~nm}$ and data signal light at $1554 \mathrm{~nm}$ are placed on two sides of a zero dispersion wavelength of the HNLF. This in turn can further alleviate a walk-off between sampling and signal pulses or a phase mismatch due to GVD, and therefore, can improve the temporal resolution of the designed waveform sampling gate. In the experiment, the average powers of sampling and data signal lights are measured to be $-4 \mathrm{dBm}$ and $1 \mathrm{dBm}$ at the HNLF input, respectively. When the phase-matching condition is satisfied, the FWM effect happens in the HNLF and leads to the generation of a sampling product (i.e., idler) at a new wavelength $\lambda_{\text {idler }}=c / v_{\text {idler }}$, where $c$ is the velocity of light in free space and $v_{\text {idler }}$ is the optical frequency of an idler wave governed by Eq. (2). In this way, the FWM

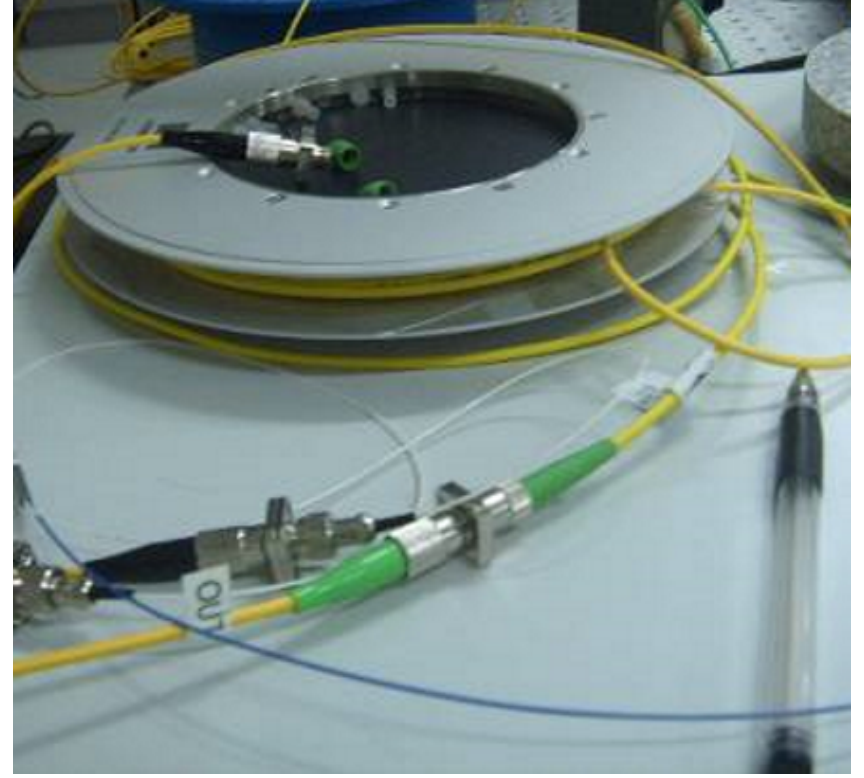

Fig. 7. A reel of $100 \mathrm{~m}$-long highly nonlinear fibre used in the waveform-sampling experiment.

sampling process generates an idler wave which carries the series of discrete-time optical samples (at rate $f_{s}$ and wavelength $\lambda_{\text {idler }}$ ) of the optical signal under test, as conceptually illustrated in Fig. 2(a) (3). Then, a tunable OBPF with $3 \mathrm{~dB}$ bandwidth of $1 \mathrm{~nm}$ is employed to filter out the idler by tuning the central wavelength of this OBPF to $\lambda_{\text {idler }}=1534 \mathrm{~nm}$. Figure 8 shows the optical spectra measured at the outputs of HNLF and OBPF 2, respectively. Since the filtered idler contains the sampled optical short pulses having a relatively low pulse rate of about $50 \mathrm{MHz}$ and a very low duty cycle, this can result in the impulse response from a low-bandwidth (i.e., 200 $\mathrm{MHz}$ ), low-noise photodetector (PD) with high linearity when the idler is photodetected. In this case, the amplitudes of output electronic pulses are proportional to the optical peak powers of the corresponding discrete-time optical samples input to the PD, so that the envelope of series of the electronic pulses will be used subsequently to reconstruct the waveform of an original optical signal under test. In practice, the detected signal at the PD output can be weak, which needs to be linearly amplified by a low-noise electronic amplifier in order to facilitate the high-precision signal processing at the electronic A/D converter.

The output of a low-bandwidth photodetector is an analogue electronic signal which cannot be processed by a conventional personal computer. For the purpose of digital signal processing and waveform/eye-diagram measurement, an A/D converter with sampling rate of $400 \mathrm{MHz}$ and resolution of 12 bits, which is controlled by a personal computer in our designed optical sampling system, is used to sample the analogue electronic signal after photodetection. Then, the result- 


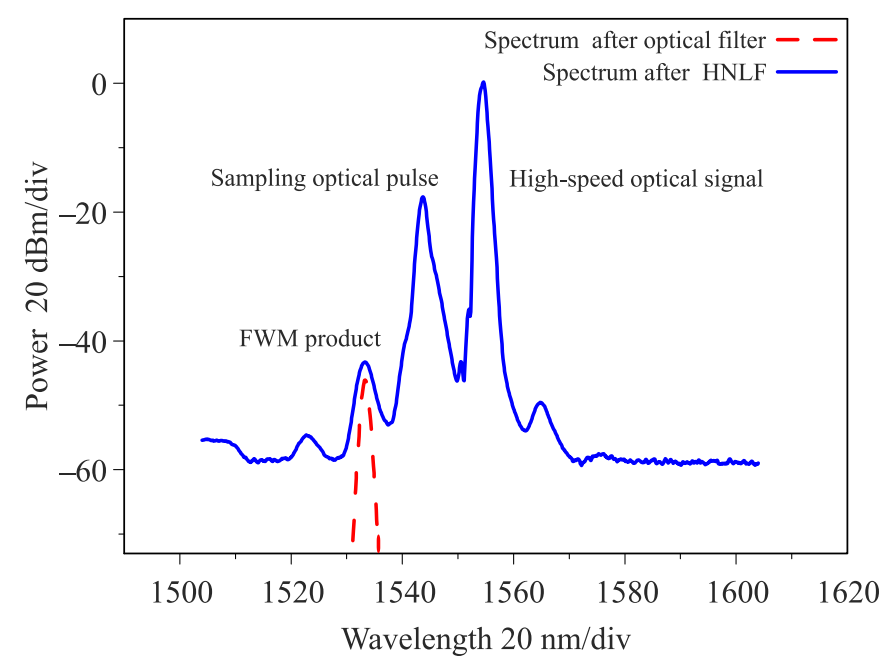

Fig. 8. Optical spectra at the outputs of HNLF and OBPF 2, respectively.

ing electronic data are fed into a personal computer for further processing. Since the reconstruction of data signal waveform and eye diagram requires the synchronization information on the acquired samples, a conventional approach for achieving this aim is to employ hardware clock recovery and trigger circuit. However, doing so can significantly increase the complexity and cost of an optical waveform sampling system in ultrahigh-speed optical data communications, which also reduces the operation flexibility of such waveform sampling systems due to the bit-rate dependence of a hardware clock recovery circuit. To solve these problems, Westlund et al. demonstrated the first software-synchronized all-optical sampling system which can achieve the synchronization of the sampled data in high precision by using only the synchronization algorithm based on the Fourier transform of the sampled data and the eye-diagram timing drift $[1,10,11]$. Therefore, we adopt this cost-effective software-synchronization method in the design of our optical waveform sampling system to facilitate the use of a free-running mode-locked fibre laser with tunable repetition rate, leading to the reduction of system complexity by avoiding any hardware clock recovery circuit. In this way, the asynchronously acquired sample data after A/D conversion are analysed by a personal computer using a digital signal processing technique in order to extract the synchronization information and to recover the timebase for equivalent-time optical waveform sampling of ultrashort optical pulses or optical data pulse signals. Consequently, the number of the scanned bit slots, $S$, is obtained from the truncated sample data of length $N$ by means of appropriate digital signal processing (e.g., Fourier transform of the truncated sample data with suitable length and subsequent determination of accurate time positions corresponding to the transition between mark and space levels of the optical signal eye diagram). For the given bit period $T_{b}=1 / B$, a time step $\Delta T$ for scanning the bit slot is substantially computed according to the relationship $\Delta T=S \cdot T_{b} / N$. For instance, $\Delta T$ can be accurately obtained by employing a high-precision iteration based on the smallest eye-diagram timing drift $[10,11]$.

Based on the software-synchronized optical sampling technique [10,11], we have developed a computer algorithm for use in our constructed optical waveform sampling system to display the optical waveforms/eye diagrams on a computer screen. In this way, the computer can correctly position a series of the asynchronously acquired samples in the time axis according to the computed $\Delta T$ and the number of consecutive bit slots required for waveform display. With the software-synchronized timebase, the signal waveform of an optical pulse stream or the eye diagram of an optical data signal under test is thus reproduced by the personal computer with the correct time scale obtained from $\Delta T$. The associated pulse parameters can be also assessed by measuring the reconstructed optical signal waveform (or eye diagram). To demonstrate the waveform sampling of ultrashort optical pulses, we start with the measurement of a $10 \mathrm{GHz}$ optical clock pulse sequence in our experiments. After photodetection and A/D conversion, a computer is employed to first reproduce a series of raw samples without correct time scale when the waveform of a $10 \mathrm{GHz}$ optical clock pulse sequence is optically sampled at the HNLF-based waveform sampling gate, as shown in Fig. 9(a). Clearly, the envelope of those acquired discrete samples contains a certain number of pulse clusters shown on the computer screen, which is analogous to the waveform of the original optical signal except for the time scale. Each cluster consists of multiple pulses with proper peaks corresponding to the impulse response of a low-bandwidth photodetector module, as illustrated in Fig. 9(b). By using the accurately estimated $\Delta T$, the curve fitting of such a pulse cluster results in the reconstruction of a single pulse waveform with correct time scale for the $10 \mathrm{GHz}$ optical clock pulse sequence under test [see Fig. 9(c)]. Our designed waveform sampling system shows that the width, $\tau_{\text {measure }}$, of the optical pulse under test is $2.0 \mathrm{ps}$. In this way, the waveform of a $10 \mathrm{GHz}$ optical clock pulse sequence is correctly rebuilt and is clearly displayed on the computer screen with 4 optical pulses of very low duty cycle, as shown in Fig. 10. Note that both optical clock and data-signal pulses employed in our experiment have an original pulse width, $\tau_{\text {original }}$, of 1.8 ps (see Fig. 5) measured by a high-resolution autocorrelator. The temporal resolution, $t_{r e}$, of an optical waveform sampling system can be computed by using the following formula [1][2]

$$
t_{r e}=\sqrt{\tau_{\text {measure }}^{2}-\tau_{\text {original }}^{2}} .
$$

Therefore, this results in $t_{r e}=\sqrt{2.0^{2}-1.8^{2}}=0.87 \mathrm{ps}$ for our constructed waveform sampling system. 


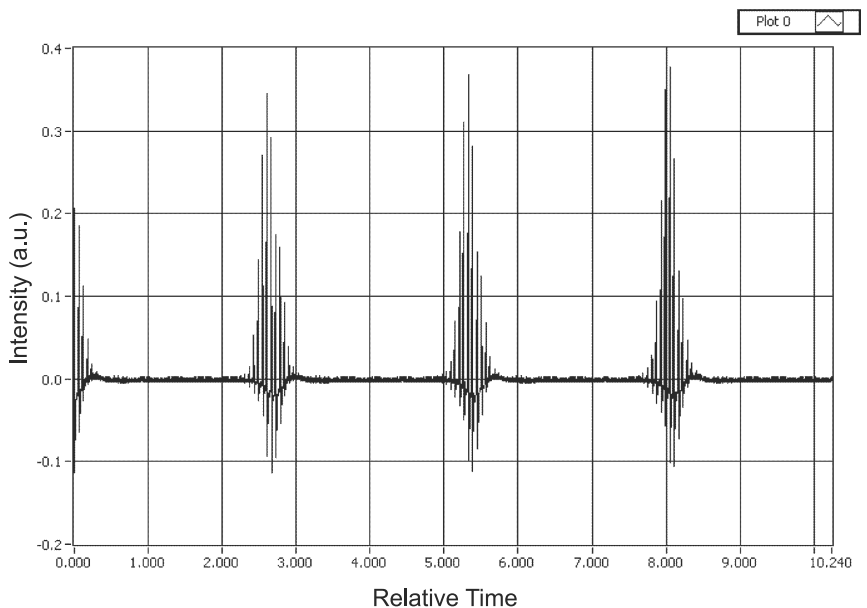

(a)

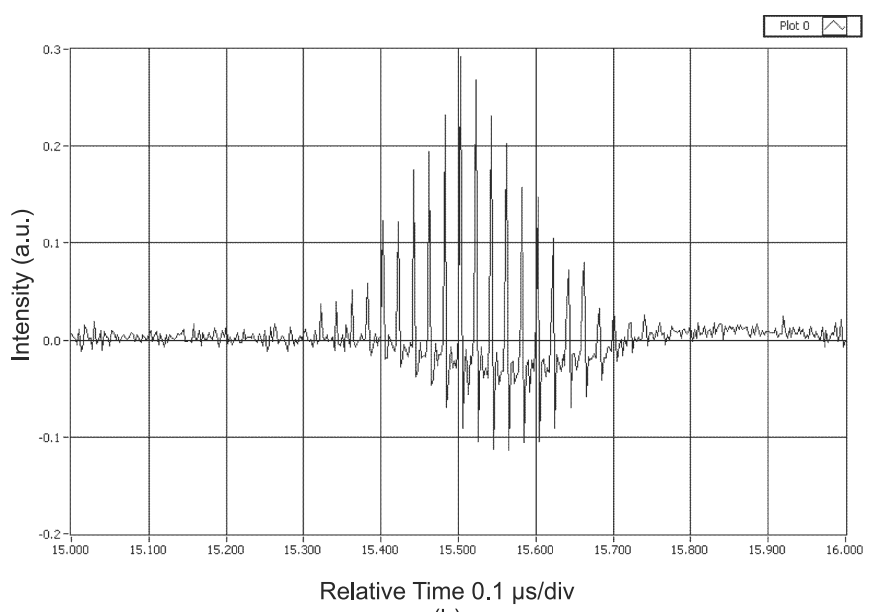

(b)

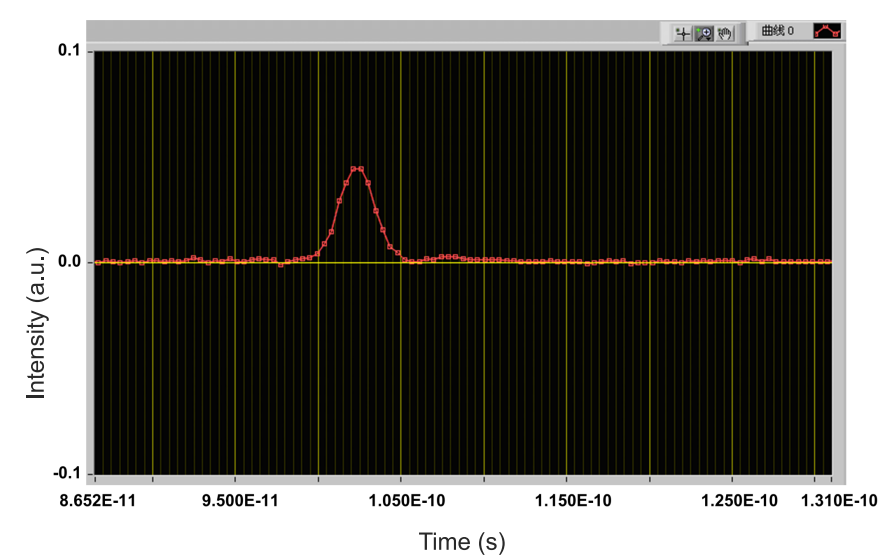

(c)

Fig. 9. Optical waveform sampling of a $10 \mathrm{GHz}$ optical clock pulse sequence. (a) The raw optical samples without correct time scale reproduced by a computer. (b) The detailed structure of a pulse cluster from raw samples. (c) The reconstructed optical pulse with correct time scale.

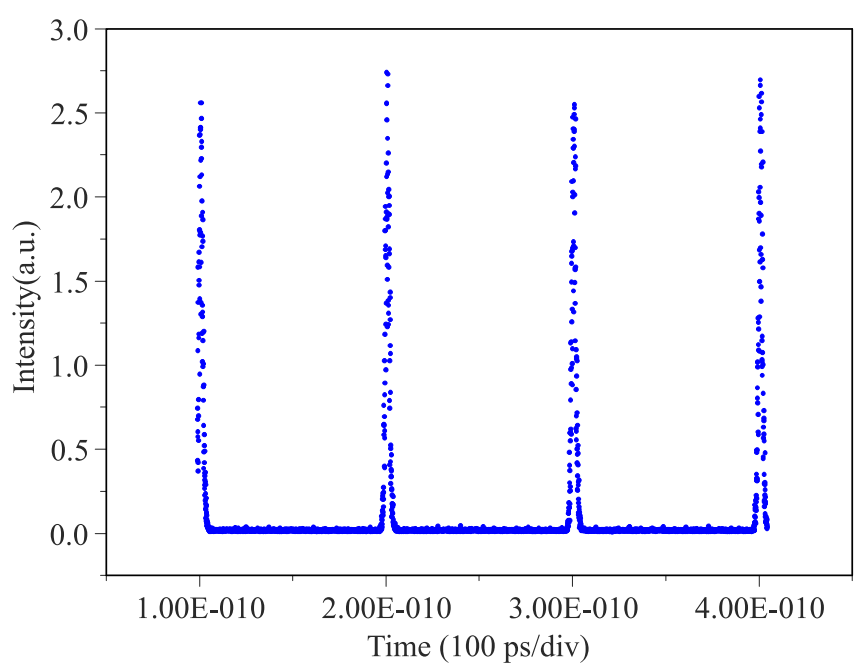

Fig. 10. Dynamic display of the measured waveform for a $10 \mathrm{GHz}$ optical clock pulse sequence with an original pulse width of $1.8 \mathrm{ps}$.

In the experiment, we can use the designed optical waveform sampling system to accurately measure the pulse shapes of a $10 \mathrm{Gbit} / \mathrm{s}$ optical PRBS data and a $80 \mathrm{Gbit} / \mathrm{s}$ OTDM signals produced by the optical pulse signal generators (see Fig. 4), respectively. The reproduced eye diagram of the $10 \mathrm{Gbit} / \mathrm{s}$ optical data signal is shown in Fig. 11, which opens widely and clearly. Our designed optical waveform sampling system can be used to flexibly display the waveform of the $80 \mathrm{Gbit} / \mathrm{s}$ OTDM signal with a return-to-zero (RZ) format and a repeated pulse pattern of " 11110000 " used in the experiment. For example, this may be practically required by some users to characterize the waveform of such an OTDM signal in the relatively long observation duration to meet some of their specific measurement needs, as illustrated in Figs. 12(a) and 12(b). Moreover, the displayed opti-

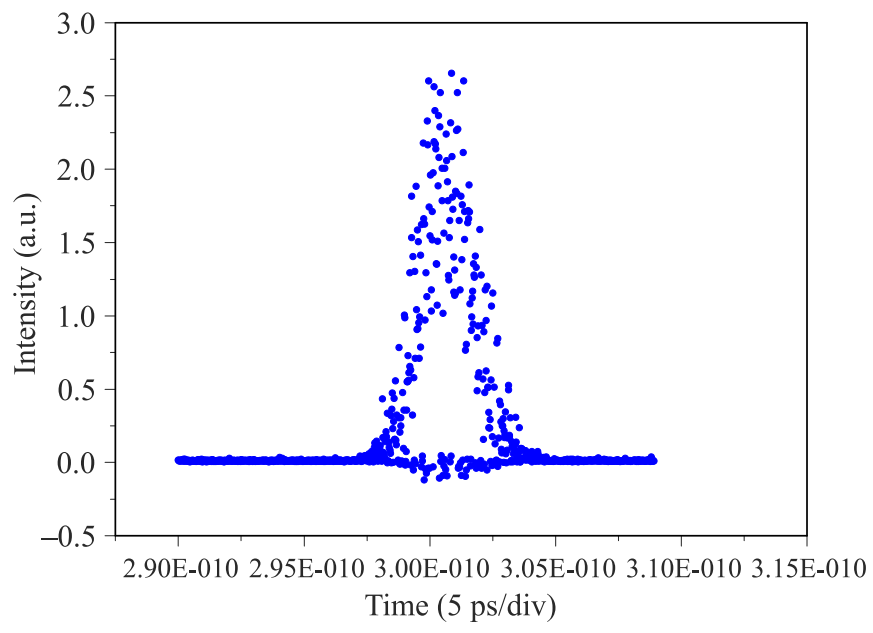

Fig. 11. The reproduced eye diagram of a $10 \mathrm{Gbit} / \mathrm{s}$ optical PRBS data signal. 


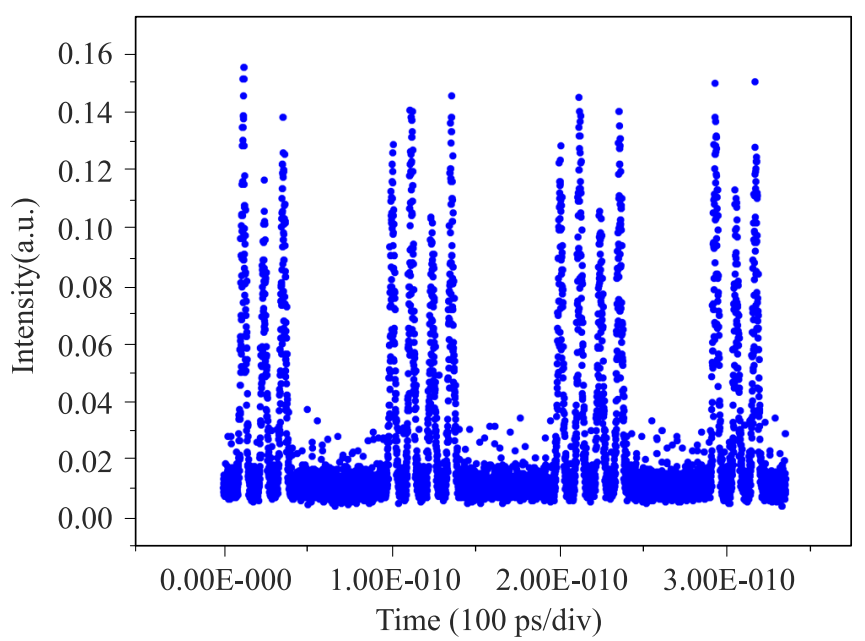

(a)

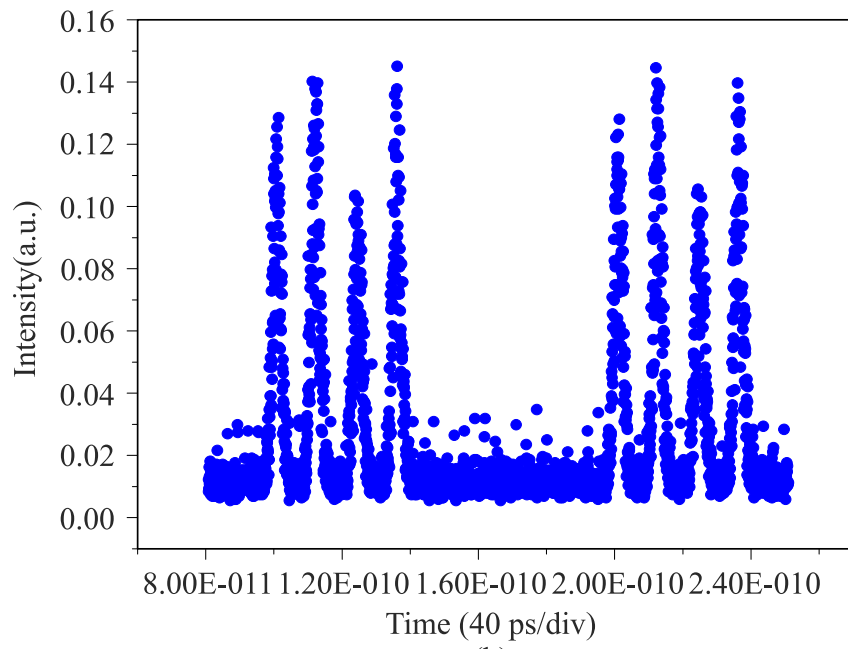

(b)

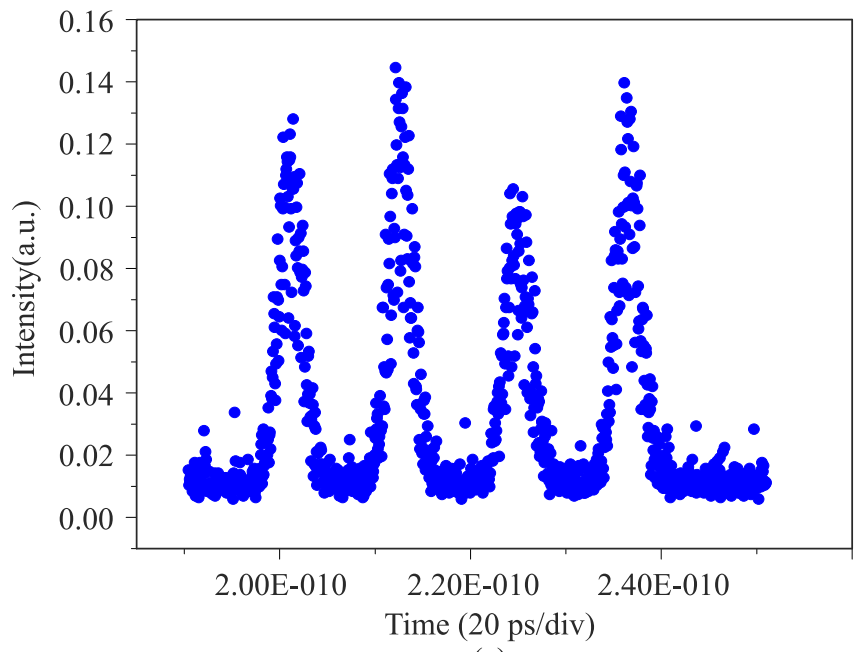

(c)

Fig. 12. The measured waveform of a $80 \mathrm{Gbit} / \mathrm{s}$ OTDM RZ signal with repeated data pattern of " 11110000 ". (a) and (b) Displays of a full pattern "11110000" in relatively long observation durations. (c) Display of a zoom-in pulse pattern "1111". cal signal waveform can be easily zoomed in with a correct time scale shown on the computer screen (e.g., see Figs. 12(b) and 12(c). In doing so, this allows us to more clearly observe a specific part or pulse pattern of the optical signal under test, as shown in Fig. 12(c). Due to a high time resolution, our designed waveform sampling system does not cause any problem associated with measurement bandwidth for the $80 \mathrm{Gbit} / \mathrm{s}$ optical data signal under test, so that the intersymbol interference (ISI) is not observed in the reproduced waveform of such a RZ signal with $\tau_{\text {original }}=1.8$ ps. In fact, the bit period of a $80 \mathrm{Gbit} / \mathrm{s}$ data signal is $12.5 \mathrm{ps}$ (i.e., the pulse spacing for a data pattern of "1111"). This in turn guarantees a clear separation between two adjacent optical pulses with the measured pulse width of only $2.0 \mathrm{ps}$ in the "1111" pulse pattern, and therefore, the reproduced waveform shows that there is no overlap between neighbouring optical pulses. In contrast, if we use a conventional optoelectronic waveform monitoring system consisting of a $70 \mathrm{GHz}$ electronic sampling oscilloscope and a $70 \mathrm{GHz}$ photodiode, the severe ISI effect would be easily observed from the measured 80 Gbit/s signal waveform as illustrated in Fig. 6. Moreover, the high resolution of our designed optical waveform sampling system allows us to inspect the detail of each RZ pulse in the measured optical signal waveform with a repeated "11110000" data pattern. For example, we can easily see that the third optical pulse clearly has the lowest peak among four successive pulses and the peak of the first pulse is moderate compared with those of the remaining three pulses as illustrated in Fig. 12, whereas it is not easy to find out such pulse details from the $80 \mathrm{Gbit} / \mathrm{s}$ signal waveform measured by employing a conventional optoelectronic waveform monitoring system due to the limited measurement bandwidth. From both Figs. 6 and 12, the uneven pulse amplitudes of this optical "11110000" data pattern are also observed, which is caused by an imperfect OTDM multiplexer having slightly unequal power losses in four optical paths [see Fig. 4(b)]. In our experiment, we have used the discrete optical couplers, tunable optical delay lines, a variable optical attenuator, and SMF pigtails to implement a bulk OTDM multiplexer. As a result, it is difficult to keep identical power losses for all the optical paths in such a multiplexer. This problem could be solved if we will be able to use an integrated waveguide OTDM multiplexer in the future. Furthermore, the quality of the displayed waveforms for both $80 \mathrm{Gbit} / \mathrm{s}$ and $10 \mathrm{Gbit} / \mathrm{s}$ optical data signals can be feasibly improved when a high-sensitivity, low-noise optical receiver with appropriate bandwidth is designed and is used in our constructed optical waveform sampling system to optimize the signal-to-noise ratio for an analogue electronic signal after photodetection. This topic will need to be studied further and will be reported separately. 


\section{Conclusions}

Optical sampling based on ultrafast optical nonlinearities is a useful technique to measure the waveforms of ultrashort optical pulses and to monitor the eye diagrams of ultrahigh-speed optical data signals. This goal has been achieved by adopting both fibre-based optical hardware implementation and software-synchronized optical sampling technique [10, 11]. Using our constructed free-running passively mode-locked femtosecond fibre laser of tunable repetition rate and low timing jitter as a high-performance optical sampling pulse source, we have presented a new implementation of optical waveform sampling systems in this paper. The designed optical sampling system is based on FWM effect in a 100 m-long HNLF for ultrafast optical signal waveform sampling and our developed computer algorithm for optical waveform display and measurement based on the software-synchronized optical sampling technique, respectively. We have successfully demonstrated the all-optical waveform sampling of a $10 \mathrm{GHz}$ optical clock pulse sequence and a $80 \mathrm{Gbit} / \mathrm{s}$ optical data signal with pulse width of $1.8 \mathrm{ps}$. Since the waveforms of the tested optical pulse signals have been accurately reproduced with a pulse width of $2.0 \mathrm{ps}$, a temporal resolution of $0.87 \mathrm{ps}$ has been achieved for optical waveform measurement in the experiment. We have also used our developed optical sampling system to accurately measure the optical eye diagram of a 10 Gbit/s optical data signal which was based on 1.8ps optical pulses and was modulated with a pseudorandom bit sequence. It is expected that optical waveform sampling systems will play an important role in fast and accurate characterization of ultrashort optical pulses, as well as future ultrahigh-speed all-optical communication systems and networks.

\section{Acknowledgement}

This research work is supported in part by the Natural Science Foundation of China (\#61275164) and is also supported by the Chinese Academy of Sciences (CAS) through a project of the CAS/SAFEA International Partnership Program for Creative Research Teams. The authors thank Prof. Wei Zhao and Prof. Guofu Chen of the State Key Laboratory of Transient Optics and Photonics at Xi'an Institute of Optics and Precision Mechanics in China for help.

\section{References}

1. P.A. Andrekson and M. Westlund, "Nonlinear optical fibre based high resolution all-optical waveform sampling", Laser \& Photon. Rev. 1, 231-248 (2007).

2. M. Westlund, P.A. Andrekson, H. Sunnerud, J. Hansryd, and J. $\mathrm{Li}$, "High-performance optical-fibre-nonlinearity-based optical waveform monitoring", J. Lightwave Technol. 23, 2012-2022 (2005).
3. M. Shirane, Y. Hashimoto, H. Yamada, and H. Yokoyama, "A compact optical sampling measurement system using mode-locked laser-diode modules", IEEE Photon. Technol. Lett. 12, 1537-1539 (2000).

4. R.L. Jungerman, G. Lee, O. Buccafusca, Y. Kaneko, N. Itagaki, R. Shioda, A. Harada, Y. Nihei and G. Sucha, "1-THz bandwidth C- and L-band optical sampling with a bit rate agile timebase", IEEE Photon. Technol. Lett. 14, 1148-1150 (2002).

5. N. Yamada, S. Nogiwa, and H. Ohta, "640-Gb/s OTDM signal measurement with high-resolution optical sampling system using wavelength-tunable soliton pulses", IEEE Photon. Technol. Lett. 16, 1125-1127 (2004).

6. C. Schmidt-Langhorst, C. Schubert, C. Boerner, V. Marembert, S. Ferber, R. Ludwig, and H.G. Weber, "Optical sampling system including clock recovery for $320 \mathrm{Gbit} / \mathrm{s}$ DPSK and OOK data signals", Proc. Optical Fibre Commun. Conf., Paper OWJ6, Anaheim, California, 2005.

7. J. Li, M. Westlund, H. Sunnerud, B.E. Olsson, M. Karlsson, and P.A. Andrekson, " $0.5-\mathrm{Tb} / \mathrm{s}$ eye-diagram measurement by optical sampling using XPM-induced wavelength shifting in highly nonlinear fibre", IEEE Photon. Technol. Lett. 16, 566-568 (2004).

8. I. Shake, E. Otani, H. Takara, K. Uchiyama, Y. Yamabayashi, and T. Morioka, "Bit rate flexible quality monitoring of 10 to $160 \mathrm{Gbit} / \mathrm{s}$ optical signals based on optical sampling technique", Electron. Lett. 36, 2087-2088 (2000).

9. C. Schmidt-Langhorst, C. Schubert, C. Boerner and R. Ludwig, "Optical sampling technologies and applications", Proc. Opt. Fibre Commun. Conf. Paper OTuG2, Anaheim, California, 2005.

10. M. Westlund, H. Sunnerud, M. Karlsson, and P.A. Andrekson, "Software-synchronized all-optical sampling for fibre communication systems", J. Lightwave Technol. 23, 1088-1099 (2005).

11. M. Westlund, H. Sunnerud, M. Karlsson, and P. Andrekson, "Software-synchronized all-optical sampling", Proc. Opt. Fibre Commun. Conf. pp. 409-410, Atlanta, 2003.

12. H. Ji, H. Hu, M. Galili, L.K. Oxenlowe, M. Pu, K. Yvind, J.M. Hvam, and P. Jeppesen, "Optical waveform sampling and error-free demultiplexing of $1.28 \mathrm{Tbit} / \mathrm{s}$ serial data in a silicon nanowire", Proc. Opt. Fibre Commun. Conf., Paper PDPC7, San Diego, 2010.

13. J. Van Erps, F. Luan, M.D. Pelusi, T. Iredale, S. Madden, D.-Y. Choi, D.A. Bulla, B. Luther-Davies, H. Thienpont, and B.J. Eggleton, "High-resolution optical sampling of $640-\mathrm{Gb} / \mathrm{s}$ data using four-wave mixing in dispersion-engineered highly nonlinear $\mathrm{As}_{2} \mathrm{~S}_{3}$ planar waveguides", J. Lightwave Technol. 28, 209-215 (2010).

14. H. Ohta, N. Banjo, N. Yamada, S. Nogiwa, and Y. Yanagisawa, "Measuring eye diagram of $320 \mathrm{Gbit} / \mathrm{s}$ optical signal by optical sampling using passively modelocked fibre laser", Electron. Lett. 37, 1541-1542 (2001).

15. H. Takara, S. Kawanishi, T. Morioka, K. Mori, and M. Saruwatari, "100Gbit/s optical waveform measurement with $0.6 \mathrm{ps}$ resolution optical sampling using subpicosecond supercontinuum pulses", Electron. Lett. 30, 1152-1153 (1994).

16. T. Kiatchanog, K. Igarashi, T. Tanemura, D. Wang, K. Katoh, and K. Kikuchi, "Real-time all-optical waveform sampling using a free-running passively mode-locked fibre laser as the 
Optical waveform monitoring based on a free-running mode-locked femtosecond fibre laser and four-wave mixing...

sampling pulse source", Proc. Opt. Fibre Commun. Conf., Paper OWN1, Anaheim, CA, 2006.

17. I. Kang and K.F. Dreyer, "Sensitive 320 Gbit/s eye diagram measurements via optical sampling with semiconductor optical amplifier-ultrafast nonlinear interferometer", Electron. Lett. 39, 1081-1083 (2003).

18. Y. Liu, J.-G. Zhang, and W. Zhao, "Design of wideband, high-resolution optical waveform samplers based on a dispersion-flattened highly nonlinear photonic crystal fibre", J. Opt. 14, 055201 (9pp), (2012).

19. A. Otani, Y. Tsuda, K. Igawa, and K. Shida, "Novel optical sampling oscilloscope using envelope detection triggering method”, J. Lightwave Technol. 26, 2991-998 (2008).
20. Y.S. Liu and J.-G. Zhang, "Design of low timing-jitter, stable picosecond optical-pulse source by using an uncooled gain-switched Fabry-Perot semiconductor laser with external continuous-wave light injection", Microwave Opt. Technol. Lett. 53, 2100-2105 (2011).

21. Y. Liu, J.-G. Zhang, G. Chen, W. Zhao, and J. Bai, "Low-timing-jitter, stretched-pulse passively mode-locked fibre laser with tunable repetition rate and high operation stability", $J$. Opt. 12, 095204 (2010).

22. D. Tang, J.-G. Zhang, and Y. Liu, "Highly stable, passively mode-locked fibre laser with low pump power for subpicosecond pulse generation", Laser Phys. 22, 1586-1589 (2012). 